\title{
Case Report \\ Hyper IgG4-Related Disease Presenting with Orbital Tumor and Immune Deficiency
}

\author{
Caroline G. Olson $\mathbb{D i D}^{1}$ and Nancy Y. Olson $\mathbb{D}^{2}$ \\ ${ }^{1}$ University of Missouri-Kansas City, School of Medicine, Kansas City, MO, USA \\ ${ }^{2}$ Allergy and Rheumatology Clinic of Kansas City, Overland Park, KS, USA \\ Correspondence should be addressed to Caroline G. Olson; carolinegolson@gmail.com
}

Received 2 July 2021; Accepted 2 September 2021; Published 20 September 2021

Academic Editor: Ahmad Mansour

Copyright ( $) 2021$ Caroline G. Olson and Nancy Y. Olson. This is an open access article distributed under the Creative Commons Attribution License, which permits unrestricted use, distribution, and reproduction in any medium, provided the original work is properly cited.

\begin{abstract}
We report a case of IgG4-RD in a patient with high IgG4 levels, low functional antibodies, and low IgM levels. He presented with bilateral orbital pseudotumors and, after initial improvement on corticosteroids, relapsed with recurrent pleural effusion and pelvic pseudotumor. He had a grossly elevated serum IgG $(1905 \mathrm{mg} / \mathrm{dl})$ with elevations in all IgG subclasses but marked elevation in IgG4 (412 mg/dl), low IgM, and low pneumococcal antibodies. Orbital mass biopsy showed polyclonal lymphocytic infiltration and increased IgG4 plasma cells. The patient was started on prednisone and tried several immunosuppressive medications including mycophenolate mofetil, methotrexate, hydroxychloroquine, and azathioprine with decrease in size of the orbital pseudotumor. During a period when the patient stopped his medications, the pseudotumor enlarged with new development of recurrent pleural effusions. He was also found to have a pelvic mass that was biopsy positive for IgG4 proliferation. This case with progression to multiorgan involvement highlights the importance of identifying patients with IgG4-related disease. In contrast to previous cases with normal-to-high IgM, the IgM was low with impaired functional antibodies.
\end{abstract}

\section{Introduction}

Immunoglobulin G4-related disease (IgG4-RD) is a rare relapsing-remitting fibroinflammatory disease caused by monoclonal proliferation of IgG4+ plasma cells and dense lymphoplasmacytic infiltration with reversible collagen deposition [1-3]. It typically presents with insidious onset in middle-aged to elderly men, although studies have shown higher IgG4 levels and worse disease in Asian populations $[1,3-5]$. IgG4+ plasma cells produce antibodies that may regulate the immune system and protect against hypersensitivity anaphylaxis in patients with allergies $[6,7]$. $\mathrm{Pa}-$ tients often have a longstanding history of allergic rhinitis, bronchial asthma, or eczema at diagnosis. IgG4- RD can be asymptomatic or present with symptoms of mechanical compression exerted by pseudotumor-like fibrotic masses. The most common manifestations have been grouped into four clinical phenotypes including pancreatobiliary (type 1 autoimmune pancreatitis (AIP)), retroperitoneal fibrosis and aortitis, head and neck limited, and systemic with salivary or lacrimal gland swelling (Mikulicz disease) $[1,8-10]$. One study found that IgG4 played a role in all archival cases of idiopathic retroperitoneal fibrosis analyzed [4]. Atypical presentations including tubulointerstitial nephritis, glomerulonephritis, perineural disease, prostatitis, orchitis, sclerosing mastitis, eosinophilic angiocentric fibrosis, interstitial lung disease, and pleural and pericardial effusions have been reported $[1,11,12]$. Intrathoracic involvement can include parenchymal opacities and interstitial lung disease, tracheobronchial stenosis, fibrosing mediastinitis, nodular pleural lesions, and rarely, pleural effusion and may resemble sarcoidosis or lung cancer [3,13]. Salivary and lacrimal gland involvement can cause facial and orbital swelling and can present similarly to Sjogren's syndrome with sclerosing sialadenitis [1, 14]. Orbital pseudotumors involve the lacrimal gland in $69 \%$ of cases but can also occur in extraocular muscles, palpebrae, optic nerve, and orbital bone, as well causing exophthalmos and restriction of ocular 
movements [1, 15-17]. These orbital manifestations tend to have higher relapse rates on steroids and overall have higher cumulative doses of corticosteroids [10]. Several studies have shown a link between lacrimal gland involvement and development of lymphoma [18]. The 2019 ACR/EULAR standards for diagnosis of IgG4-RD are based on the presence of characteristic clinical or radiological involvement of a typical organ or pathological evidence of an inflammatory lymphoplasmacytic infiltrate of uncertain etiology. In addition, the patients must not have another explanation for their symptoms including infection, autoimmune disease, or malignancy. Thirdly, there is a grading scale with points awarded for histopathology with dense lymphoplasmacytic infiltrate and storiform fibrosis with or without obliterative phlebitis, positive immunostaining, serum IgG4 $>5 \mathrm{x}$ the upper limit of normal, having a set of glands involved, and having pulmonary or pancreas findings, kidney findings, and retroperitoneal findings [19]. In $10-20 \%$ of cases, antinuclear antibodies (ANAs) are also positive [4]. Cases of IgG4-RD have been described with elevated or normal levels of IgM, but low IgM and low functional antibodies have not previously been reported.

\section{Case Presentation}

The patient was a 66-year-old Caucasian male with class III obesity (BMI 42) and a history of adult-onset asthma and allergies referred by his ophthalmologist for bilateral orbital masses that showed IgG4 lymphoproliferative disease on biopsy. On the right side, the medial superior orbital mass was approximately $1.0 \mathrm{~cm} \times 1.3 \mathrm{~cm}$ and left-sided mass was $4 \mathrm{~mm} \times 4 \mathrm{~mm}$. A biopsy of both right and left eyelid lesions including the bone and inferior orbital fat pad showed polyclonal lymphocytic infiltration and increased IgG4 plasma cells up to $98 / \mathrm{HPF}$. The majority were $\mathrm{T}$ cells with a nonspecific mild increase in CD4:CD8 ratio and normal antigen expression. B cells were polyclonal with normal antigen expression. There was no immunophenotypic evidence of non-Hodgkin lymphoma. The diagnosis of IgG4 lymphoproliferative disease was given based on elevated IgG4 levels and pathologic findings, and he was referred to rheumatology. Systemic review was positive for fatigue, easy bruising and bleeding, cough, shortness of air, wheezing, hypertension, joint pain, red dry eyes, and postnasal drip. He did not smoke, denied recurrent infection other than rhinitis, and had no pertinent family history.

CT of the chest showed no evidence of pulmonary parenchymal fibrosis or interstitial lung disease. Laboratory investigations at the initial visit showed elevated IgG with extremely elevated IgG4, decreased functional antibodies, and low IgM. Total IgG $1905 \mathrm{mg} / \mathrm{dl}$ (694-1618 mg/dl), IgG1 $1106 \mathrm{mg} / \mathrm{dl}$ (382-929 mg/dl), IgG2 $598 \mathrm{mg} / \mathrm{dl}$ (241-700 mg/ dl), IgG3 $98 \mathrm{mg} / \mathrm{dl}(22-178 \mathrm{mg} / \mathrm{dl})$, and IgG4 $412 \mathrm{mg} / \mathrm{dl}$ $(4-86 \mathrm{mg} / \mathrm{dl})$. This gives an IgG4/IgG ratio of .216 which correlates with values $\geq 0.2$ in patients with IgG4-RD. IgA was normal at 222, and IgM was 40 . His initial pneumococcal vaccination was in three years prior to presentation.
Three of 14 pneumococcal antibody titers were at a protective level $(>1.3 \mathrm{mcg} / \mathrm{ml})$. Haemophilus influenza antibodies were decreased at 0.34. Angiotensin-converting enzyme (ACE) was low. Rheumatoid factor was positive at 28, and cyclic citrullinated peptide (CCP) was negative.

The patient was started on prednisone $20 \mathrm{mg}$ daily, and to decrease steroid need with his weight, mycophenolate mofetil $1000 \mathrm{mg}$ BID was added. Mycophenolate was discontinued due to side effects of dizziness and nausea. He was dissatisfied with steroid weight gain at prednisone $20 \mathrm{mg}$ daily (BMI had increased to 44.9). On this regimen, he had marked improvement in asthma symptoms and reduced size of orbital pseudotumors. To continue to try to achieve corticosteroid sparing, he briefly used methotrexate and then added hydroxychloroquine as an adjunctive treatment with no further change in tumor size, so he was unwilling to continue them. Pneumovax booster and one Prevnar booster dose were administered while the patient was off immunosuppression. An additional Prevnar booster was given while he was on low-dose prednisone. He had an increase in pneumococcal protective antibodies from 3 of 14 to 4 of 14 during treatment. When the larger right orbital pseudotumor had regressed, the patient preferred to stop taking the prednisone and declined treatment for a period of 18 months. During this time, his bilateral eye lesions returned to near baseline size before treatment. Laboratory studies at this time showed an eosinophil count of 1096 (normal $<500)$, SPEP with increased protein, decreased $\operatorname{IgM}$ at $20 \mathrm{mg} / \mathrm{dl}$ (normal $>48 \mathrm{mg} / \mathrm{dl}$ ), IgG $3570 \mathrm{mg} / \mathrm{dl}$ (normal $<1618 \mathrm{mg} / \mathrm{dl}$ ), IgG4 $1594 \mathrm{mg} / \mathrm{dl}$ (normal <86 mg/dl), IgG4/ IgG ratio of 0.45 , ESR of $104 \mathrm{~mm} / \mathrm{h}$ (normal $<20 \mathrm{~mm} / \mathrm{h}$ ), and elevated CRP at $9.9 \mathrm{mg} / \mathrm{dl}$ (normal $<8.0 \mathrm{mg} / \mathrm{dl}$ ) (See Table 1). The patient also began to report increased shortness of breath, and chest X-ray showed large left pleural effusion (Figure 1). Thoracentesis yielded $2 \mathrm{~L}$ of fluid from the left lung that was negative for malignant cells but showed the presence of reactive mesothelial cells and histiocytes. He saw cardiology and had workup for pleural effusion including neoplastic and infectious causes.

The patient was unwilling to take corticosteroids again because of the efforts he had made to lose weight. He was willing to try azathioprine. He had a recurrence of leftsided pleural effusion, and repeat thoracentesis showed similar findings. He underwent pleurodesis on the left thorax. He was started on azathioprine $50 \mathrm{mg}$ BID and corticosteroids, and during this period, the right orbital pseudotumor was at its smallest appearance. At this time, the patient was following with oncology and the CT/PET scan showed $3.3 \mathrm{~cm} \times 1.9 \mathrm{~cm}$ mass in his pelvis near the left seminal vesicle as well as incidental liver lesion without PET scan uptake. Biopsy of the pelvic mass showed IgG4 stain strongly positive. Oncology started prednisone $80 \mathrm{mg}$ daily with taper, and the size of the pelvic mass decreased in size to $2.1 \mathrm{~cm} \times 1.1 \mathrm{~cm}$. The patient continues to undergo ongoing treatment with intermittent steroids and azathioprine. Current pneumococcal antibody levels after a total of two Prevnar and two Pneumovax booster doses are 7 out of 14 protective. 
TABLE 1: Laboratory values at presentation, several months into treatment while on corticosteroids and mycophenolate mofetil, and during relapse.

\begin{tabular}{|c|c|c|c|c|c|c|c|c|c|}
\hline & $\begin{array}{c}\text { IgG total } \\
\text { (nl 694-1618 mg/ } \\
\mathrm{dl} \text { ) }\end{array}$ & $\begin{array}{c}\text { IgG4 } \\
\text { (nl } 4-86 \mathrm{mg} / \\
\mathrm{dl} \text { ) }\end{array}$ & $\begin{array}{c}\operatorname{IgM} \\
(\mathrm{nl}>48 \mathrm{mg} / \\
\mathrm{dl})\end{array}$ & $\begin{array}{c}\text { ESR } \\
(\mathrm{nl}<20 \mathrm{~mm} / \\
\mathrm{hr})\end{array}$ & $\begin{array}{c}\text { CRP } \\
(\mathrm{nl}<8 \mathrm{mg} / \\
\mathrm{dl})\end{array}$ & $\begin{array}{c}\text { Pneumococcal } \\
\text { antibodies } \\
(\mathrm{nl}>8 / 14)\end{array}$ & $\begin{array}{l}\text { Haemophilus } \\
\text { antibodies } \\
(\mathrm{nl}>1)\end{array}$ & $\begin{array}{l}\text { Platelets } \\
\text { (nl } \\
150-450)\end{array}$ & $\begin{array}{l}\text { Eosinophils } \\
\text { (nl 15-500) }\end{array}$ \\
\hline Presentation & 1905 & 412 & 44 & 19 & 0.25 & $2 / 14$ & 0.26 & 258 & 17 \\
\hline $\begin{array}{l}\text { During } \\
\text { treatment }\end{array}$ & 1760 & 377 & 43 & 11 & 1.09 & $3 / 14$ & 0.34 & 240 & 432 \\
\hline Relapse & 3570 & 1594 & 20 & 104 & 9.3 & $4 / 14$ & 0.17 & 250 & 1096 \\
\hline
\end{tabular}

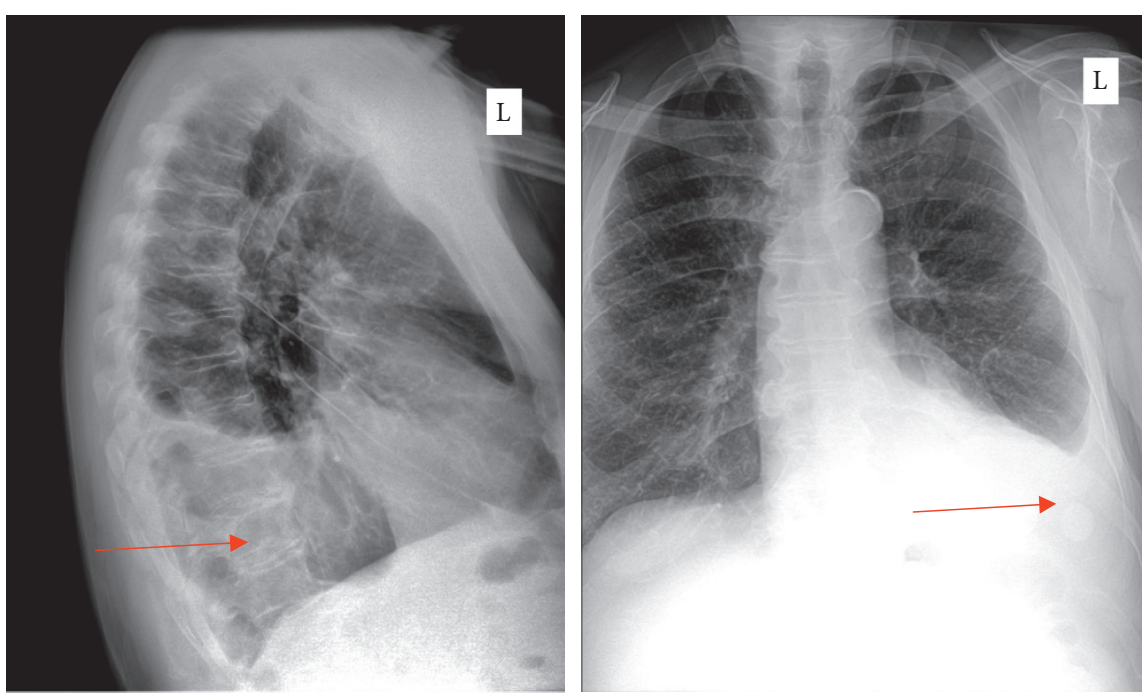

Figure 1: Lateral and posterior-anterior chest X-ray showing left-sided pleural effusion (arrows) during symptom relapse.

\section{Discussion}

This patient presented with hyper IgG4-related orbital pseudotumors but, over time, developed recurrent pleural effusions and a pelvic pseudotumor with notable laboratory findings including low IgM and low functional protective antibodies.

The IgG4 subclass accounts for only $3-6 \%$ of the total IgG fraction, and up to $5 \%$ of the normal healthy population may have elevated IgG4 levels $[3,6,19,20]$. IgG4 has been linked to several diseases that are clinically separate from IgG4-RD including pemphigus vulgaris, membranous glomerulonephritis, rheumatoid arthritis, Castleman syndrome, Wegener granulomatosis, pulmonary abscess, and thrombotic thrombocytopenic purpura [1, 21-23]. In IgG4related disease, the hallmark is a lymphoplasmacytic infiltrate mainly consisting of IgG4+ plasma cells along with characteristic histological findings. The role of IgG4 plasma cells is not clearly understood as studies have not provided insight into this process. CD19, CD27, and CD38+ plasma blasts, as well as clonally expanded CD4+ and CD8+ cytotoxic $\mathrm{T}$ lymphocytes, have been found to be elevated in the blood and in fibrotic lesions of patients with IgG4-RD $[2,24,25]$. One study showed a significant presence of M2 macrophages among collagen fibers in fibrotic areas [26]. Cytokines thought to be involved in this process include IL13 and TGF-B in fibroblast activation, as well as IL- 4 and IL10 in class switching of IgG to IgG4 [2]. A Japanese study has identified several loci that indicated increased susceptibility for development of IgG4-RD including HLA-DRB1 and FCy [27]. Serological findings in IgG4-RD are nonspecific but can include elevations in erythrocyte sedimentation rate (ESR) and CRP, as well as positive ANA in $50 \%$ of patients and elevated RF in 20\% [28]. Eosinophilia with increased IgE has been found in $30-50 \%$ of IgG4-RD lesions, and an eosinophilic predominance can be seen in orbital or upper respiratory tract disease $[1,21]$. Typical serum protein electrophoresis shows polyclonal hypergammaglobulinemia with beta-gamma bridging [5]. Serum IgG4 levels above $1.35 \mathrm{~g} / \mathrm{dl}$ may indicate higher risk for type 1 AIP and IgG4related sclerosing cholangitis and can be used to differentiate pancreatic cancer from sclerosing cholangitis [20, 29, 30]. It may be useful to monitor serial IgG4 levels or circulating plasmablasts to guide treatment, but evidence is still unclear $[1,11,31]$. Two histological findings are needed for diagnosis including whorly storiform fibrosis, obliterative phlebitis, dense lymphoplasmacytic infiltrate with many IgG4+ plasma cells, and eosinophilic infiltrate or granuloma formation $[1,6,17,23]$.

In IgG4-related disease, IgM, IgA, and other IgG subclasses are frequently elevated [1]. In contrast to the current literature, our patient presented with persistently suppressed IgM levels and low protective antibody levels. In healthy individuals, IgM autoantibodies play an important role in modulating immune responses and clearing self-antigens [32-34]. IgM deficiency typically presents with repeated infections but may also have an increased rate of allergic and autoimmune disease than the general population $[32,34]$. 
Almost $40 \%$ of patients with IgM deficiency present with asthma and allergic rhinitis [32]. One study showed that patients with IgM deficiency had a higher rate of pathogenic ANA and $14 \%$ developed autoimmune disease [35]. Hashimoto's thyroiditis, SLE, multiple myositis, myasthenia gravis, and Addison's disease have been linked to low IgM levels $[32,36]$. Although this patient did not have significant sinopulmonary infections, he may have been at risk for autoimmune disease related to immune deficiency. In IgM deficiency, cases of IgG subclass deficiency have been reported, but hyper IgG4 disease has been reported only in one previous case report [34, 36, 37]. Immune response to vaccination has not been mentioned in IgG4-related disease. Some patients with selective IgM deficiency or IgG subclass deficiency have been shown to have impaired IgG responses to the pneumococcal polysaccharide vaccines which may improve with immunoglobulin therapy $[34,38]$. At presentation, this patient was positive for only 3 of 14 protective pneumococcal antibodies as well as low haemophilus protective antibodies. His response to Pneumovax was poor. Repeated pneumococcal vaccines both Pneumovax and Prevnar were required to maintain his protective antibodies, and overall, he had a much weaker than expected polysaccharide response, only increasing from 3 to 4 protective antibodies after vaccination.

Treatment of IgG4-RD typically consists of prednisolone $0.6 \mathrm{mg} / \mathrm{kg} / \mathrm{d}$ with gradual taper over a period of 3-6 months [39]. Patients with elevated IgG4 at baseline, multiorgan involvement, and history of disease relapse may have a higher tendency to relapse after steroids have been discontinued. As a result, glucocorticoid sparing treatments have been used including azathioprine, mycophenolate mofetil, methotrexate, cyclophosphamide, and tacrolimus, but studies have not been conducted to compare efficacies of these agents [39]. Glucocorticoids with immunosuppressants have been shown to have a higher relapse-free rate than glucocorticoids alone [16]. In patients with refractory disease, rituximab can decrease serum IgG4 subclass concentrations. Early treatment is more likely to improve disease manifestations in patients with prominent lymphoplasmacytic infiltrate and especially in glandular tissues [1, 40]. When disease progresses to tightly organized collagen bundles, definitive treatment is less successful, but potential to reverse collagen formation is still present. In this patient, the smallest tumor size was achieved with glucocorticoid taper. Glucocorticoids also caused weight gain, so the patient was reluctant to restart them after losing weight, so several glucocorticoid-sparing treatments were tried in conjunction, with the most symptom improvement during treatment with mycophenolate mofetil and azathioprine. His case was complicated by poor adherence to treatment due to medication side effects and lack of concern on his part once the orbital lesions decreased in size.

Several case studies have described incidence of immune thrombocytopenia in patients with IgG4 disease. The incidence of this finding is unknown but has been postulated to be caused by antiplatelet antibodies or other IgG subclasses of platelet antibody [41]. This patient had normal platelet levels throughout presentation and treatment. Isolated pleural effusion has been reported in several case studies of IgG4-related disease, but it is much more commonly seen with other lung manifestations such as parenchymal or pleural lesions $[8,42]$.

This case report highlights and demonstrates the relative effectiveness of steroid-sparing medications in IgG4-RD treatment, and shows a rare presentation of IgG4-RD with low IgM levels and low functional antibodies.

\section{Data Availability}

No data were used to support this study other than patient medical record.

\section{Consent}

Written informed consent was obtained from the patient to publish this case report and any accompanying images.

\section{Conflicts of Interest}

The authors declare no conflicts of interest regarding the publication of this article.

\section{References}

[1] E. Della-Torre, M. Lanzillotta, and C. Doglioni, "Immunology of IgG4-related disease," Clinical \& Experimental Immunology, vol. 181, no. 2, pp. 191-206, 2015.

[2] D. Michailidou and P. Cohen, IgG4-Related Kidney Disease: Diagnostics, Manifestations \& More, The Rheumatologist, vol. 12, no. 5, pp. 17-20, 2018.

[3] J. H. Ryu, H. Sekiguchi, and E. S. Yi, "Pulmonary manifestations of immunoglobulin G4-related sclerosing disease," European Respiratory Journal, vol. 39, no. 1, pp. 180-186, 2012.

[4] G. H. Neild, M. Rodriguez-Justo, C. Wall, and J. O. Connolly, "Hyper-IgG4 disease: report and characterisation of a new disease," BMC Medicine, vol. 4, no. 1, p. 23, 2006.

[5] L. Y. C. Chen, A. Mattman, M. A. Seidman, and M. N. Carruthers, "IgG4-related disease: what a hematologist needs to know," Haematologica, vol. 104, no. 3, pp. 444-455, 2019.

[6] Y. Zen, S. Kitagawa, H. Minato et al., "IgG4-positive plasma cells in inflammatory pseudotumor (plasma cell granuloma) of the lung," Human Pathology, vol. 36, no. 7, pp. 710-717, 2005.

[7] D. C. Trampert, L. M. Hubers, S. F. J. van de Graaf, and U. Beuers, "On the role of IgG4 in inflammatory conditions: lessons for IgG4-related disease," Biochimica et Biophysica Acta (BBA) - Molecular Basis of Disease, vol. 1864, no. 4, pp. 1401-1409, 2018.

[8] S. N. Campbell, E. Rubio, and A. L. Loschner, "Clinical review of pulmonary manifestations of IgG4-related disease," Annals of the American Thoracic Society, vol. 11, no. 9, pp. 1466-1475, 2014.

[9] M. Takahira, M. Kawano, Y. Zen, H. Minato, K. Yamada, and K. Sugiyama, "IgG4-Related chronic sclerosing dacryoadenitis," Archives of Ophthalmology, vol. 125, no. 11, pp. 1575-1578, 2007.

[10] M. Lanzillotta, C. Campochiaro, G. Mancuso et al., "Clinical phenotypes of IgG4-related disease reflect different prognostic outcomes," Rheumatology, vol. 59, no. 9, pp. 2435-2442, 2020. 
[11] H. Pieringer, I. Parzer, A. Wöhrer, P. Reis, B. Oppl, and J. Zwerina, "IgG4- related disease: an orphan disease with many faces," Orphanet Journal of Rare Diseases, vol. 9, no. 1, p. 110, 2014.

[12] H. Yamamoto, M. Yasuo, Y. Nomura et al., "IgG4-related airway involvement which developed in a patient receiving corticosteroid therapy for autoimmune pancreatitis," Internal Medicine, vol. 50, no. 24, pp. 3023-3026, 2011.

[13] K. Tsushima, T. Tanabe, H. Yamamoto et al., "Pulmonary involvement of autoimmune pancreatitis," European Journal of Clinical Investigation, vol. 39, no. 8, pp. 714-722, 2009.

[14] T. Kamisawa, H. Nakajima, N. Egawa, N. Funata, K. Tsuruta, and A. Okamoto, "IgG4-related sclerosing disease incorporating sclerosing pancreatitis, cholangitis, sialadenitis and retroperitoneal fibrosis with lymphadenopathy," Pancreatology, vol. 6, no. 1-2, pp. 132-137, 2006.

[15] A. Shenoy, N. Mohandas, and A. Gottlieb, "Cutaneous and systemic IgG4-related disease: a review for dermatologists," Dermatology Online Journal, vol. 25, no. 6, https:// escholarship.org/uc/item/9w91m8dz, 2019.

[16] Z. Zhao, D. Mou, Z. Wang et al., "Clinical features and relapse risks of IgG4-related ophthalmic disease: a single-center experience in China," Arthritis Research \& Therapy, vol. 23, no. 1, p. 98, 2021.

[17] T. Kubota and S. Moritani, "Orbital IgG4-related disease: clinical features and diagnosis," ISRN Rheumatology, vol. 2012, Article ID 412896, 5 pages, 2012.

[18] J. A. Plaza, J. A. Garrity, A. Dogan, A. Ananthamurthy, T. E. Witzig, and D. R. Salomão, "Orbital inflammation with IgG4-positive plasma cells," Archives of Ophthalmology, vol. 129, no. 4, pp. 421-428, 2011.

[19] Z. S. Wallace, R. P. Naden, S. Chari et al., "American college of rheumatology/European league against rheumatism IgG4related disease classification criteria working group. The 2019 American college of rheumatology/European league against rheumatism classification criteria for IgG4-related disease," Arthritis \& Rheumatism, vol. 72, no. 1, pp. 7-19, 2020.

[20] H. Hamano, S. Kawa, A. Horiuchi et al., "High serum IgG4 concentrations in patients with sclerosing pancreatitis," New England Journal of Medicine, vol. 344, no. 10, pp. 732-738, 2001.

[21] J. H. Stone, Y. Zen, and V. Deshpande, "IgG4-related disease," New England Journal of Medicine, vol. 366, no. 6, pp. 539-551, 2012.

[22] D. Inoue, Y. Zen, H. Abo et al., "Immunoglobulin G4-related lung disease: CT findings with pathologic correlations," $R a$ diology, vol. 251, no. 1, pp. 260-270, 2009.

[23] V. Deshpande, Y. Zen, J. K. Chan et al., "Consensus statement on the pathology of IgG4-related disease," Modern Pathology, vol. 25, no. 9, pp. 1181-1192, 2012.

[24] T. Kamisawa, N. Funata, Y. Hayashi et al., "A new clinicopathological entity of IgG4-related autoimmune disease," Journal of Gastroenterology, vol. 38, no. 10, pp. 982-984, 2003.

[25] J. Backhus, C. Neumann, L. Perkhofer et al., "A follow-up study of a European IgG4-related disease cohort treated with Rituximab," Journal of Clinical Medicine, vol. 10, no. 6, p. 1329, 2021.

[26] K. Miyabe, Y. Zen, L. D. Cornell et al., "Gastrointestinal and extra-intestinal manifestations of IgG4-related disease," Gastroenterology, vol. 155, no. 4, pp. 990-1003, 2018.

[27] M. Lanzillotta, G. Mancuso, and E. Della-Torre, "Advances in the diagnosis and management of IgG4 related disease," $B M J$, vol. 369 , p. m1067, 2020.
[28] S. Nambiar and T. I. Oliver, IgG4 Related DiseaseStatPearls Publishing, 2021, https://www.ncbi.nlm.nih.gov/books/ NBK499825/.

[29] M. Ebbo, A. Grados, E. Bernit et al., "Pathologies associated with serum IgG4 elevation," The Internet Journal of Rheumatology, vol. 2012, Article ID 602809, 2012.

[30] T. Saeki, A. Saito, H. Yamazaki et al., "Tubulointerstitial nephritis associated with IgG4-related systemic disease," Clinical and Experimental Nephrology, vol. 11, no. 2, pp. 168-173, 2007.

[31] M. N. Carruthers, A. Khosroshahi, T. Augustin, V. Deshpande, and J. H. Stone, "The diagnostic utility of serum IgG4 concentrations in IgG4-related disease," Annals of the Rheumatic Diseases, vol. 74, no. 1, pp. 14-18, 2015

[32] S. Gupta and A. Gupta, "Selective IgM deficiency-an underestimated primary immunodeficiency," Frontiers in Immunology, vol. 8, p. 1056, 2017.

[33] C. Campochiaro, S. Atay, K. E. N. Clark, V. Ong, and C. P. Denton, "Autoimmunity and immunodeficiency at the crossroad: autoimmune disorders as the presenting feature of selective IgM deficiency," BMJ Case Reports, vol. 12, no. 1, Article ID e223180, 2019.

[34] A. G. Louis and S. Gupta, "Primary selective IgM deficiency: an ignored immunodeficiency," Clinical Reviews in Allergy \& Immunology, vol. 46, no. 2, pp. 104-111, 2014.

[35] T. T. T. Nguyen and N. Baumgarth, "Natural IgM and the development of B cell-mediated autoimmune diseases," Critical Reviews in Immunology, vol. 36, no. 2, pp. 163-177, 2016.

[36] G. Ideura, K. Agematsu, Y. Komatsu et al., "Selective IgM deficiency accompanied with IgG4 deficiency, dermal complications and a bronchial polyp," Allergology International, vol. 57, no. 1, pp. 99-105, 2008.

[37] V. Y. Y. Xu, M. Bell, A. Zahirieh et al., "A challenging diagnosis of IgG4-related disease when understanding limitations of laboratory testing was pivotal," Case reports in medicine, vol. 2017, Article ID 8748696, 5 pages, 2017.

[38] A. R. Parker, M. Skold, S. Harding, J. C. Barton, L. F. Bertoli, and J. C. Barton, "Pneumococcal vaccination responses in adults with subnormal IgG subclass concentrations," BMC Immunology, vol. 20, no. 1, p. 29, 2019.

[39] P. Brito-Zerón, B. Kostov, X. Bosch, N. Acar-Denizli, M. Ramos-Casals, and J. H. Stone, "Therapeutic approach to IgG4-related disease," Medicine, vol. 95, no. 26, Article ID e4002, 2016.

[40] Y. Shimizu, M. Yamamoto, Y. Naishiro et al., "Necessity of early intervention for IgG4-related disease--delayed treatment induces fibrosis progression," Rheumatology, vol. 52, no. 4, pp. 679-683, 2013.

[41] S. Li, C. Deng, J. Chen, and F. Li, Mikulicz's Disease with Immune Thrombocytopenia: A New Immunoglobulin G4Mediated Disorder and Literature Review (NEEDS Formatting) Department of Rheumatology and Immunology, Central South University, Changsha, China, 2018.

[42] J. H. Choi, J. K. Sim, J. Y. Oh et al., "A case of IgG4-related disease presenting as massive pleural effusion and thrombophlebitis," Tuberculosis and Respiratory Diseases, vol. 76, no. 4, pp. 179-183, 2014. 